\title{
Coming full circle in cancer
}

\section{identified}

several

potentially

functional

circRNAs

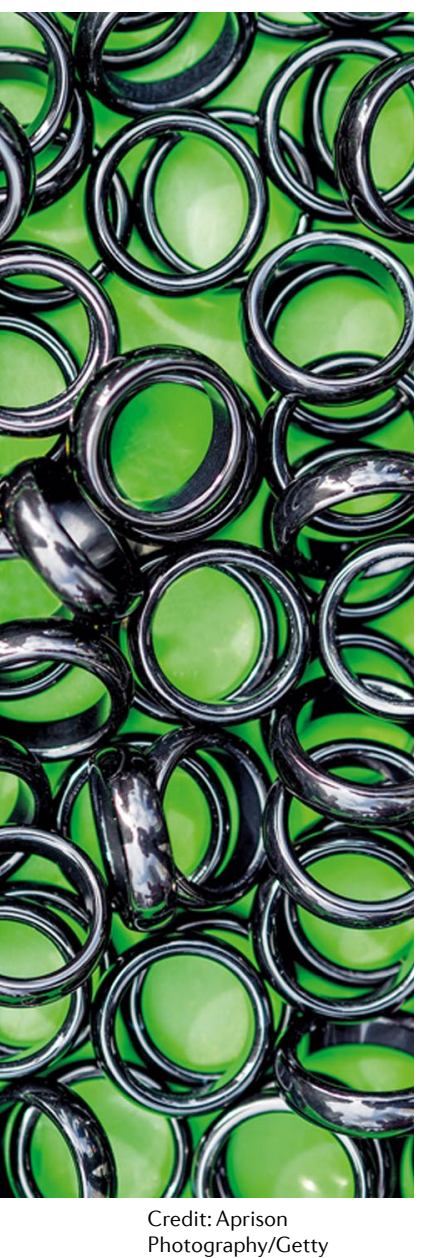

Circular RNAs (circRNAs) are singlestranded RNA circles formed from back-splicing of linear transcripts whereby the $3^{\prime}$ end of one exon is spliced to the $5^{\prime}$ end of its own or an upstream exon. Although their existence has been known for several decades, it has remained unclear how widespread or functionally relevant they are. Three new studies characterize circRNAs in cancer, with potential clinical relevance.

Most cancer RNA sequencing (RNA-seq) studies use protocols that enrich RNAs based on poly(A) tails. As circRNAs lack this feature, alternative methods are required.

The three studies used distinct clinical sample sets and RNA preparation methodologies. Vo et al. used >2,000 cancer samples and cell lines of diverse tissue types and adopted an exome-capture RNA-seq protocol. Chen et al. characterized 144 localized prostate tumours with ribosomal RNA (rRNA)-depleted RNA-seq, and Smid et al. analysed 348 primary breast tumours with rRNA-depleted RNA-seq incorporating random priming for cDNA synthesis. From the resulting RNA-seq data, the teams used distinct bioinformatics pipelines to identify circRNAs based on features such as non-linear splicing junctions. Each group reported thousands of circRNAs and validated a subset of these using quantitative reversetranscription PCR (qRT-PCR).

All three studies noted that exons involved in circRNA formation had characteristic features relative to those found in linear RNAs only, such as being flanked by longer introns that facilitate backsplicing. Additionally, expression levels of circRNAs were generally poorly correlated with the levels of linear transcripts from the same gene, implying that there is some differential regulation of circRNA versus linear RNA abundance.

Beyond these common observations, each study also had distinct focuses. Vo et al. used their data to form the MiOncoCirc database of tumour tissue circRNAs as a community resource. They also investigated readthrough circRNAs (rt-circRNAs) that consist of exons from neighbouring genes. For rt-circRNAs and regular circRNAs, some are found ubiquitously across tissues whereas others are lineagespecific and thus might serve as biomarkers to inform the tissue of origin of a cancer. However, as one potential caveat of circRNAs as cancer-specific biomarkers, Vo et al. reported that circRNAs are generally downregulated in cancer relative to normal tissue, and this effect seems to be connected to the increased proliferation rate of cancer cells, as seen when the proliferation rate was manipulated in cancer cell lines.

However, a subset of circRNAs were upregulated in cancer, and these might be highest priority to explore as cancer biomarkers.

Chen et al. quantified a circRNA index (CRI) as a measure of circRNA abundance and showed that for prostate cancer samples, extreme CRIs (first and fourth quartiles) correlated with worse patient outcomes, and this effect was replicated in an independent cohort. They also carried out a screen using short hairpin RNAs (shRNAs) separately targeting 1,300 of the most abundant circRNAs (via back-splice junctions) or their parental transcripts (via exons that are spliced out during circularization). They identified several potentially functional circRNAs for which knockdown of the circRNA but not the parental linear transcript impairs prostate cancer cell line proliferation. One example was circCSNK1G3, and mechanistic follow-up studies indicated that circCSNK1G3 interacts with the microRNA miR-181b/d, consequently downregulating expression of the $C B X 7$ tumour-suppressor gene.

As circRNAs lack free ends, they are more resistant to exonucleases than linear RNAs, and hence there is interest in exploring whether circRNAs could be informative disease-associated biomarkers in bodily fluids. As steps in this direction, Smid et al. showed a correlation between tumour circCNOT2 levels and clinical progression after subsequent aromatase inhibitor treatment and using qRT-PCR were able to detect variable circCNOT2 levels in plasma from patients with breast cancer. Furthermore, Vo et al. demonstrated that prostate cancer circRNAs could be detected in urine, either individually through qRT-PCR or more globally using their exome-capture RNA-seq approach due to its applicability to low-input RNA samples.

Overall, these studies provide valuable catalogues of circRNAs in cancer. It will be interesting to investigate the potential of circRNAs as non-invasive or minimally invasive biomarkers, including their diagnostic and prognostic value relative to biomarkers based on linear RNAs, DNA or proteins.

Darren J. Burgess

ORIGINAL ARTICLES $\vee$ o, J. N. et al. The landscape of circular RNA in cancer. Cell 176, 869-881 (2019) |Chen, S. et al. Widespread and functional RNA circularization in localized prostate cancer. Cell 176, 831-843 (2019)| Smid, M. et al. The circular RNome of primary breast cancer. Genome Res. https://doi.org/ 10.1101/gr.238121.118 (2019) FURTHER READING Szabo, L. \& Salzman, J. Detecting circular RNAs: bioinformatic and experimental challenges. Nat. Rev. Genet. 17, 679-692 (2016) 